\title{
Numerical Study on Stress Concentration Effect in Rapid Evaluation of Fatigue Limit through Temperature Evolution*
}

\author{
Hung Anh LY**, Hirotsugu INOUE*** and Yousuke IRIE ${ }^{\dagger}$ \\ ** Department of Aeronautical Engineering, HoChiMinh City University of Technology \\ 268 Ly Thuong Kiet St., Dist. 10, Ho Chi Minh City, Vietnam \\ *** Department of Mechanical and Control Engineering, Tokyo Institute of Technology \\ Mailbox i1-42, 2-12-1 O-okayama, Meguro, Tokyo 152-8552, Japan \\ $\dagger$ Panasonic Corporation \\ 1006 Kadoma, Kadoma City, Osaka 571-8506, Japan \\ E-mail: lyhunganh@hcmut.edu.vn
}

\begin{abstract}
The technique for rapid evaluation of fatigue limit using infrared thermography has been developed and paid much attention recently. However, the enhancement of reliability of this technique is demanded for practical application in industries. This study is conducted to verify the effect of stress concentration on fatigue limit evaluation through numerical simulation. Temperature evolutions of stainless steel specimens with different notches are simulated by 3D elasto-plastic finite element analysis. It has been shown that the fatigue limit evaluation based on the temperature evolution is essentially explained by plastic energy dissipation, and that the temperature evolution should be measured after a sufficiently large number of cycles so that plastic shakedown is achieved. It has been remarked that the fatigue limit is overestimated if the spatial resolution of infrared thermography is not fine enough to measure the temperature evolution at the stress concentration site.
\end{abstract}

Key words : Fatigue Limit, Energy Dissipation, Stress Concentration, Infrared Thermography, Finite Element Analysis

\section{Introduction}

The determination of fatigue limit by the traditional Wöhler method is properly a time and cost consuming task. Therefore, various techniques for rapidly evaluating the fatigue limit have been considered for more than 100 years $^{(1)}$. Among them, the technique based on the temperature evolution associated with cyclic loading of testing object is particularly studied in this paper. A brief review of this technique is given in the previous paper ${ }^{(2)}$. The essential disadvantage of this technique lies in the fact that it is less reliable than the Wöler method because the fatigue limit is evaluated only by several hundreds or thousands of loading cycles. Nevertheless, several researchers ${ }^{(3),(4)}$ proposed the use of infrared thermography for this technique about 20 years ago and it has been paid attention due to the recent development of infrared thermography. The reason can be considered by the use of infrared thermography which compensates for the disadvantage mentioned above since it provides full-field distribution of temperature evolution and hence enables detection of critical region of testing object including real engineering product. However, the applicability of this technique has not been verified very well especially from theoretical point of view.

In the previous study ${ }^{(2)}$, the authors conducted 3D elasto-plastic numerical simulation on the rapid evaluation of the fatigue limit and compared the result with experimental results obtained by the Wöhler method and thermography experiment. It has been shown that the fatigue limit evaluation based on the temperature evolution is essentially explained by plastic

*Received 17 Nov., 2011 (No. 11-0700) [DOI: 10.1299/jmmp.6.299]

Copyright (c) 2012 by JSME 


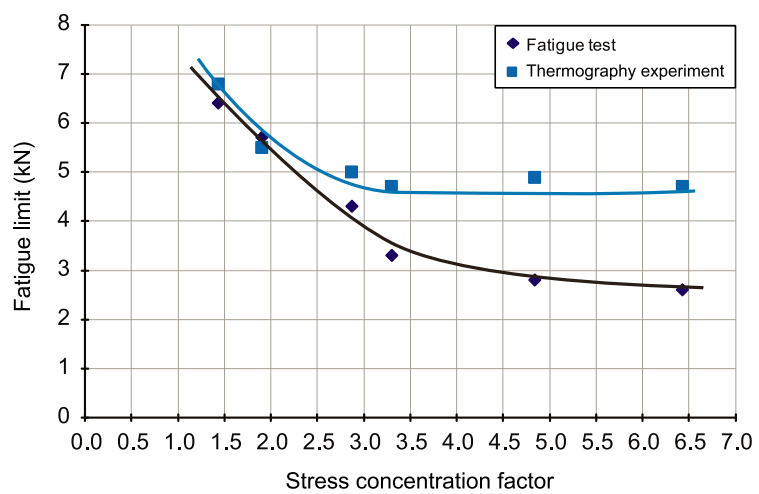

Fig. 1 Fatigue limit vs. stress concentration factors ${ }^{(5)}$.

energy dissipation. It has been emphasized that the temperature evolution should be measured after a sufficiently large number of cycles so that plastic shakedown is achieved. For the procedure determining the fatigue limit from temperature evolution data, it has been shown that $2 \mathrm{f}$-component and mean temperature rise approaches are basically the same under adiabatic condition and that the two-curve method provides better evaluation compared to the one-curve method.

However, as mentioned in the previous paper ${ }^{(2)}$ there are many issues to be studied further. In particular, the applicability of the rapid evaluation technique to stress concentration problem should be an important issue among others. Irie et al. ${ }^{(5)}$ recently considered the stress concentration effect experimentally using notched specimens with different stress concentration factors. It has been shown that the rapid evaluation technique fails to provide the fatigue limit accurately if the stress concentration factor becomes larger. Namely, the fatigue limit evaluated by the rapid technique becomes much larger than those determined by the Wöhler method when the stress concentration factor is larger than 3.0 as shown in Fig. 1. According to the result, it seems that there is some limitation in application of the rapid evaluation technique.

In this paper, 3D elasto-plastic numerical simulation is conducted for notched specimens with different stress concentration factors in order to clarify the limitation of the rapid evaluation technique.

\section{Simulation Procedure}

Numerical simulation was conducted using finite element code ABAQUS in a similar manner as in the previous paper ${ }^{(2)}$.

Geometry of the specimen used in the numerical simulation which is the same as that in the experiment ${ }^{(5)}$ is shown in Fig. 2. Only the notch radius, $R$, is varied from $5 \mathrm{~mm}$ down to $0.1 \mathrm{~mm}$ as shown in Table 1 . Stress concentration factors for these notches calculated by Heywood's equation $^{(6)}$ are shown in Table 1 . The specimen was modeled by eight-node linear reduced integration elements as shown in Fig. 3(b). Due to symmetry only one-eighth of the specimen was modeled. The number of elements is also shown in Table 1.

The material assumed was SUS304 stainless steel. A nonlinear hardening material model (Chaboche model) which was adopted to simulate ratcheting gave satisfactory results in the previous paper ${ }^{(2)}$. This model provides two features to simulate plastic hardening in cyclic loading conditions: the center of the yield surface shifts in stress space (kinematic hardening) and the size of the yield surface expands with inelastic deformation (isotropic hardening). This combination of kinematic and isotropic hardening is introduced to model the Bauschinger effect and other phenomena such as plastic shakedown, ratcheting, and relaxation of the mean stress.

The Chaboche model used in this study adopts linear back stress evolution rules of which 


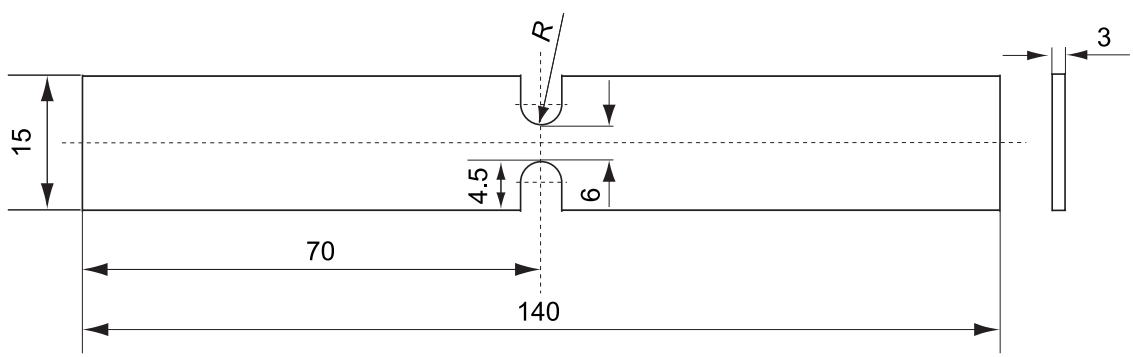

Fig. 2 Geometry of notched specimens (unit in $\mathrm{mm}$ ).

Table 1 Stress concentration factor and Number of elements

\begin{tabular}{ccccccc}
\hline Notch radius $(\mathrm{mm})$ & 5 & 2 & 0.7 & 0.5 & 0.2 & 0.1 \\
\hline Stress concentration factor & 1.43 & 1.90 & 2.87 & 3.30 & 4.84 & 6.43 \\
\hline Number of elements & 2400 & 7600 & 3880 & 3664 & 6800 & 11880 \\
\hline
\end{tabular}

evolution equation is

$$
\dot{x}=C \frac{1}{\sigma^{0}}(\sigma-x) \varepsilon^{p}-\dot{\gamma} x \dot{\varepsilon}^{p},
$$

where $x$ is translation of yield surface center with equivalent plastic strain $\varepsilon^{p}, \sigma$ is the current Cauchy stress tensor, $C$ is the initial kinematic hardening modulus, and $\gamma$ determines the rate at which the kinematic hardening modulus decreases with increasing plastic deformation. The non-linear kinematic hardening is governed by

$$
x=\frac{C}{\gamma}\left(1-e^{-\gamma \varepsilon^{p}}\right)
$$

where $C / \gamma$ is the saturated value of yield surface center translation with plastic strain. On the other hand, the isotropic hardening is governed by the yield stress during hardening

$$
\sigma^{0}=\left.\sigma\right|_{0}+Q_{\infty}\left(1-e^{-b \varepsilon^{p}}\right)
$$

where $\left.\sigma\right|_{0}$ is initial yield stress, $Q_{\infty}$ is the saturated value of increase isotropic deformation resistance and $b$ represents speed of saturation ${ }^{(7)}$. It can be also understood that $\sigma^{0}$ shows the expanding of yield surface.

Material parameters used in the numerical simulation are shown in Table 2.

The temperature evolution was computed also in the same manner as in the previous paper $^{(2)}$. Adiabatic condition was assumed for simplicity because heat transfer causes temperature evolution to be dependent on the loading rate and therefore complicated. Repeated sinusoidal stress of $\sigma_{\min } / \sigma_{\max }=0$ as shown in Fig. 3(a) was applied to the model at one end until 900 cycles while the other end was fixed in the loading direction. The stress-strain behavior at the notch root (element of interest indicated in Fig. 3(b)) was used for computing the temperature evolution of the element. The primary results obtained by the numerical analysis are the elastic strains in three coordinate axes $\varepsilon_{x x}^{e}, \varepsilon_{y y}^{e}, \varepsilon_{z z}^{e}$ and dissipated plastic energy density which corresponds to the irreversible mechanical energy. These results were used as inputs

\begin{tabular}{|c|c|c|c|c|c|}
\hline Density & $\begin{array}{l}\text { Young's } \\
\text { modulus } \\
E\end{array}$ & $\begin{array}{l}\text { Poisson's } \\
\text { ratio } \\
v\end{array}$ & $\begin{array}{c}\text { Yield } \\
\text { strength } \\
\left.\sigma\right|_{0}\end{array}$ & $\begin{array}{c}\text { Coefficient of } \\
\text { thermal expansion } \\
\alpha\end{array}$ & $\begin{array}{c}\text { Specific heat at } \\
\text { constant pressure } \\
C_{p}\end{array}$ \\
\hline $7900 \mathrm{~kg} / \mathrm{m}^{3}$ & $192 \mathrm{GPa}$ & 0.33 & $200 \mathrm{MPa}$ & $17.8 \times 10^{-6} / \mathrm{K}$ & $500 \mathrm{~J} / \mathrm{kg} . \mathrm{K}$ \\
\hline & \multicolumn{2}{|c|}{ Kinematic hardening parameters } & \multicolumn{2}{|c|}{ Isotropic hardening parameters } & \\
\hline & C & $\gamma$ & $Q_{\infty}$ & $b$ & \\
\hline & $11070 \mathrm{MPa}$ & 39.8 & $62 \mathrm{MPa}$ & 10 & \\
\hline
\end{tabular}
for data analysis procedure as follows:

Table 2 Mechanical properties of SUS304 


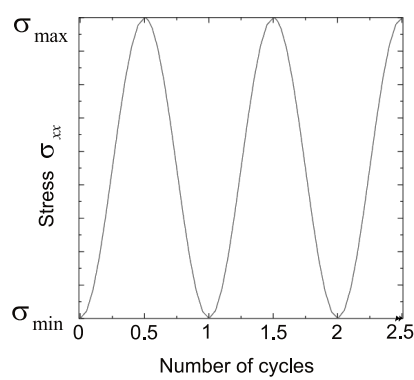

(a)

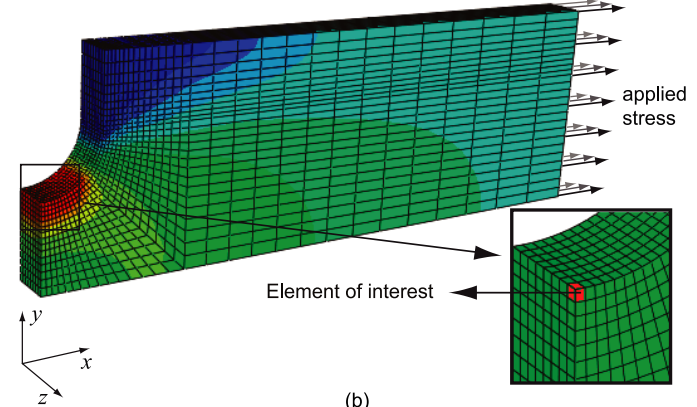

Fig. 3 (a) Cyclic stress control sinusoidal waveform.

(b) Stress distribution in $2 \mathrm{~mm}$-notched specimen at a specific time point during fatigue process and position element of interest.

( 1 ) Temperature oscillation due to the thermoelastic effect was calculated from the change in volumetric strain as

$$
\begin{aligned}
& \Delta T_{e}=\frac{-3 T \alpha K}{\rho C_{v}} \delta \varepsilon, \\
& C_{v}=C_{p}-\frac{3 \alpha^{2} T E}{\rho(1-2 v)},
\end{aligned}
$$

where $T$ is the absolute temperature, $\delta \varepsilon$ is the change in volumetric strain, $\alpha$ is the coefficient of thermal expansion, $K$ is the bulk modulus, $\rho$ is density, $C_{v}$ is the specific heat at constant volume and $C_{p}$ is the specific heat at constant pressure. Note that $\delta \varepsilon$ is independent of the co-ordinate axes in the calculation. Equation (4) is not only derived clearly in Pitarresi and Patterson $^{(8)}$ but also appeared in $\operatorname{Biot}^{(9)}$.

(2) Temperature increase due to the plastic energy dissipation, $\Delta T_{p}$, was calculated based on the dissipated energy density.

( 3 ) Temperature evolution at the interested element was obtained by combining two effects in Step (1) and Step (2) as

$$
\Delta T=\Delta T_{e}+\Delta T_{p} .
$$

\section{Results}

The rapid evaluation technique of the fatigue limit is essentially applied for engineering product in which stress distribution is often complicated and not easy to evaluate. Therefore, results of fatigue limit evaluation will be discussed in terms of applied load amplitude.

\subsection{Stress-strain behavior}

Typical stress-strain behaviors in the loading direction of the specimen at the notch tip are shown in Fig. 4, in which the loading amplitude is $6 \mathrm{kN}$ for notch radii from $5 \mathrm{~mm}$ down to $0.5 \mathrm{~mm}$ and $5 \mathrm{kN}$ for notch radii of $0.2 \mathrm{~mm}$ and $0.1 \mathrm{~mm}$. For each case, the left hand side shows stress-strain curve from the beginning to 900 cycles while the right hand side shows only five portions: 1 to 5,16 to 20,46 to 50,96 to 100 , and 600 to 900 cycles, respectively.

For every case, it is observed that the hysteresis loop is gradually shifted to right (ratcheting) and its area per one cycle decreases with the increase of the number of cycles. After a sufficient number of cycles, namely for 600 to 900 cycles, the hysterisis loop converges to a single curve (shakedown). It is noted that the number of cycles to attain the shakedown is almost the same for all cases. The area of the hysterisis loop converges to zero for 5-mm notch (Figs. 4(a1) and 4(a2)) and, therefore, no plastic energy is dissipated (elastic shakedown). For other notches, the area converges to a constant value. This is the plastic shakedown and a constant plastic energy is dissipated per one cycle. The plastic energy dissipated per one cycle after shakedown becomes larger with the decrease of the notch radius, namely the increase of the stress concentration factor. 

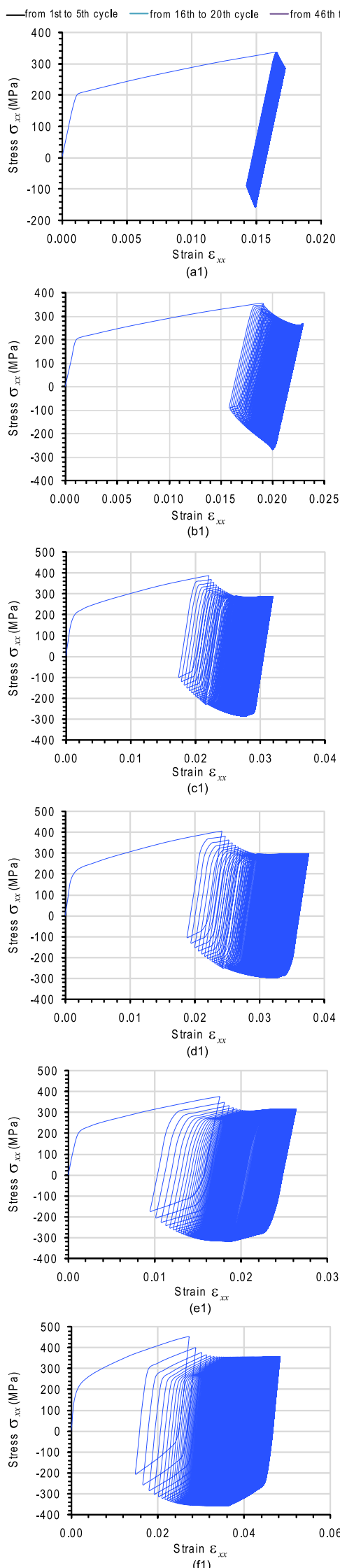
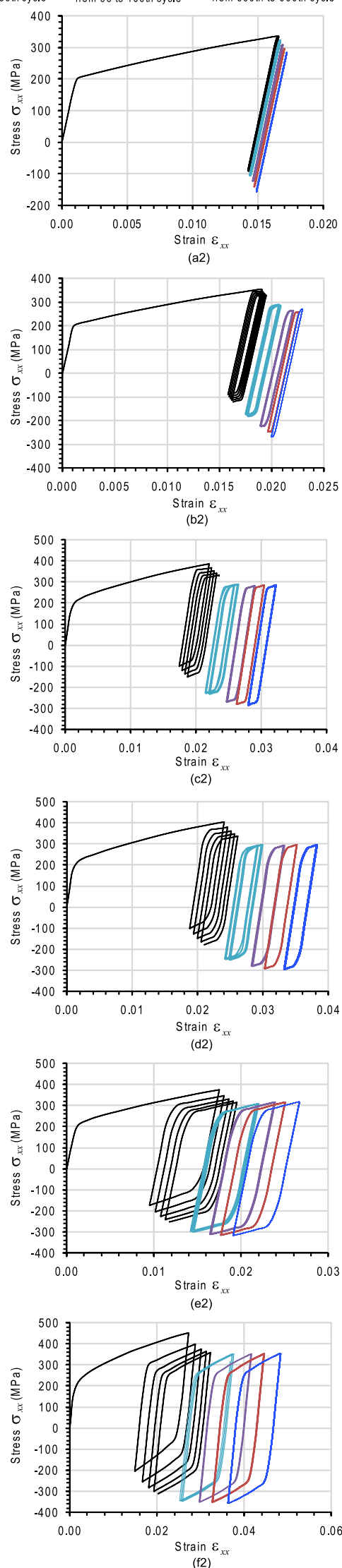

Fig. 4 Typical stress-strain curves at some cycles:

For 6-kN loading amplitude:(a1)(a2) 5mm-notch, (b1)(b2) 2mm-notch, (c1)(c2) $0.7 \mathrm{~mm}$-notch, (d1)(d2) $0.5 \mathrm{~mm}$-notch.

For 5-kN loading amplitude:(e1)(e2) $0.2 \mathrm{~mm}$-notch, (f1)(f2) $0.1 \mathrm{~mm}$-notch. 


\subsection{Temperature evolution}

Figure 5 shows temperature variation due to the thermoelastic effect at the notch tip, where the loading amplitude is $6 \mathrm{kN}$ for all notches. For each case, the left hand side shows temperature variation from the beginning to 900 cycles while the right hand side shows magnification from 400 to 405 cycles. Temperature amplitude increases with the decrease of the notch radius, which is due to stress concentration. Mean temperature increases slightly during the early cycles before reaching a saturated value. The waveform is almost sinusoidal for 5 -mm notch while it is more distorted as the notch radius decreases because yielding occurs easier due to higher stress.

Temperature increase caused by the plastic energy dissipation is shown in Fig. 6, where the loading amplitude is $6 \mathrm{kN}$ for all notches. It is observed that the temperature increases two times during one loading cycle because yielding occurs in both tensile and compressive regime. For 5-mm notch, the temperature converges to a constant value in Fig. 6(a1) after 600 cycles, which is due to the elastic shakedown as shown in Fig. 4(a1). On the other hand, for other notches, the temperature keeps increasing almost linearly. This tendency seems unreasonable but it is the consequence of the adiabatic condition assumption. The temperature should converge to a constant value for all cases if heat transfer would be taken into consideration.

The total temperature evolution is obtained as shown in Fig. 7 by combining the temperature oscillation due to the thermoelastic effect (Fig. 5) and the temperature increase due to the plastic energy dissipation (Fig. 6). For each case, the left hand side shows temperature evolution from the beginning to 900 cycles for several loading amplitudes while the right hand side shows magnification from 400 to 405 cycles for $6-\mathrm{kN}$ loading amplitude. It is understood that the temperature saturates to a constant value after 900 cycles in the cases of loading amplitude lower than $6.5 \mathrm{kN}$ for 5-mm notch and loading amplitude lower than $5.5 \mathrm{kN}$ for 2-mm notch. The temperature oscillation becomes relatively small as the plastic energy dissipation increases, which is the case when the loading amplitude becomes larger and when the notch radius becomes smaller.

\subsection{Evaluation of fatigue limit}

Two approaches called mean temperature rise approach and $2 \mathrm{f}$-component approach are known for evaluating the fatigue limit based on the temperature evolution data. The mean temperature rise approach adopted by Curti et al. ${ }^{(3)}$ and Luong ${ }^{(4)}$ is to utilize the amount of temperature rise during some specified number of cycles. On the other hand, the $2 \mathrm{f}$-component approach proposed by Brémond ${ }^{(10)}$ and applied by Krapez et al. ${ }^{(11)}$ is to utilize the amplitude of temperature evolution at the frequency twice of the loading frequency. The $2 \mathrm{f}$-component approach is based on the fact that the temperature increases twice during one loading cycle as observed in Fig. 6. In the previous paper ${ }^{(2)}$, it has been shown that these two approaches are basically equivalent each other under adiabatic condition. This fact is reexamined for different notches hereafter.

Mean temperature rise relative to the ambient temperature $\left(T_{0}=298 \mathrm{~K}\right)$ were calculated for the data shown in the left column of Fig. 7. The mean temperature rises at 100, 300, 500, 600 and 800 cycles were obtained as shown in the left column of Fig. 8. Then the mean temperature rises during every 200 cycles (consistent with experiment ${ }^{(5)}$ ) were obtained as shown in the right column of Fig. 8. For 5- and 2-mm notches, the plots converge to a line at a sufficiently large number of cycles, namely when the plastic shakedown is achieved. For other notches, the plots are always on a line. According to the conclusion of the previous paper ${ }^{(2)}$, the fatigue limit was evaluated as the loading amplitude at the intersection of the light blue line (least squares fit to the plots after the plastic shakedown) and the horizontal axis as shown in the right column of Fig. 8.

On the other hand, 2f-component amplitudes of the temperature evolution were obtained by applying FFT to every 10 cycles of the data shown in the left column of Fig. 7. The results 


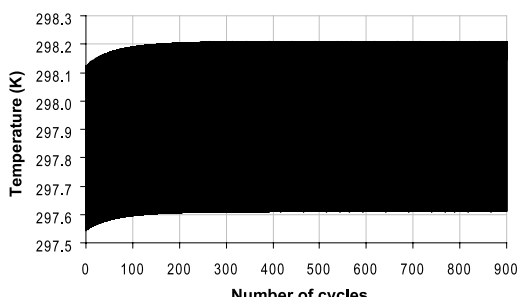

(a1)

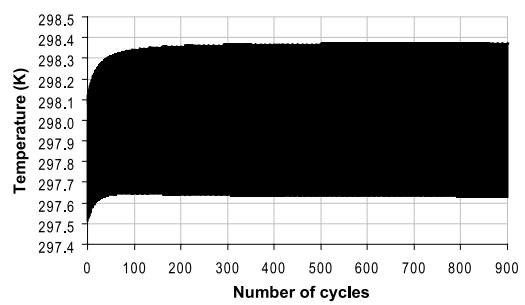

(b1)

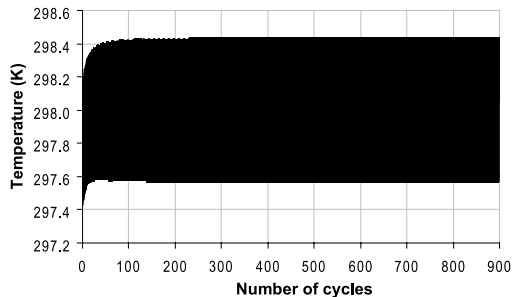

(c1)

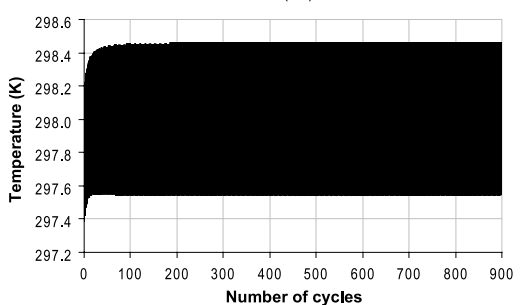

(d1)

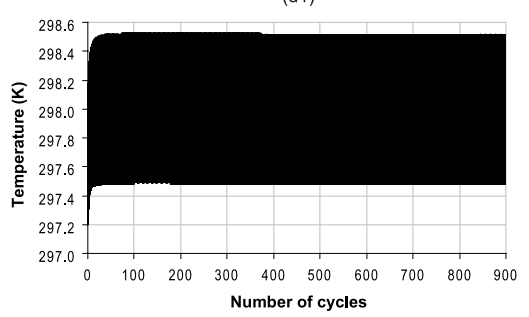

(e1)

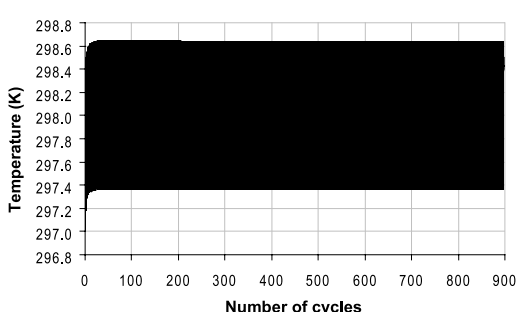

(f1)

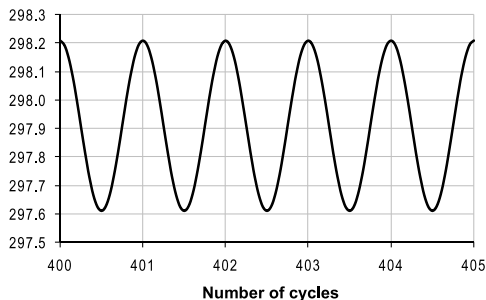

(a2)

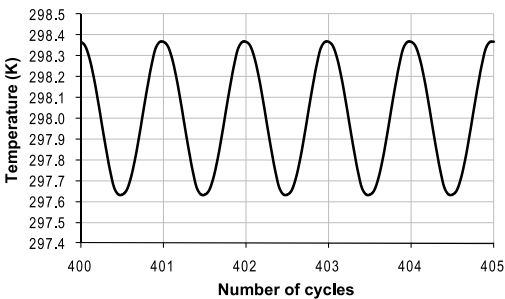

(b2)

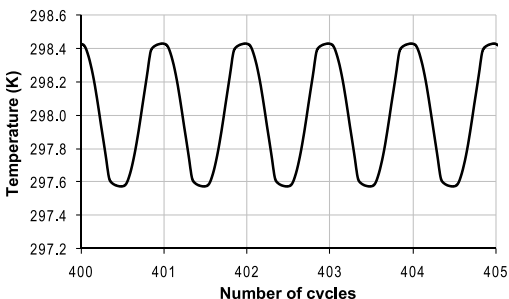

(c2)

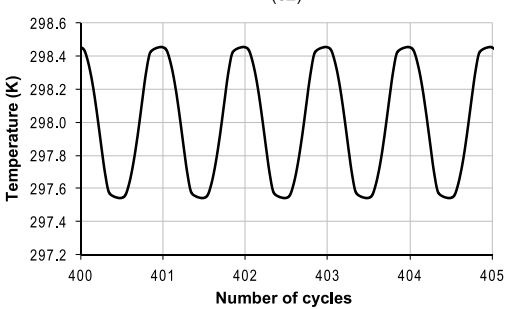

(d2)

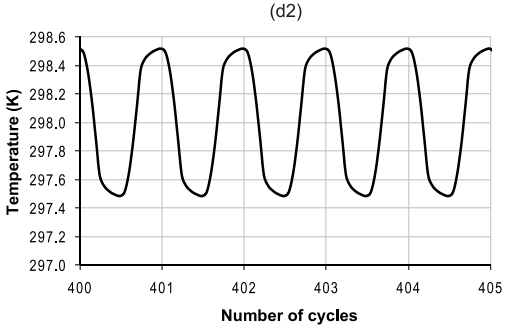

(e2)

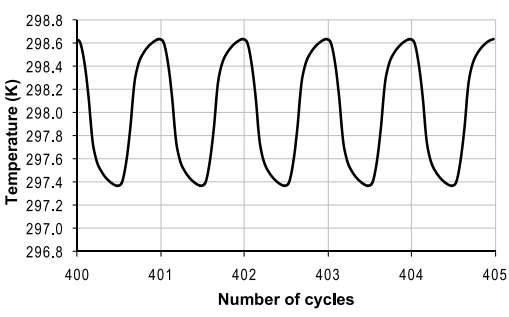

(f2)

Fig. 5 Temperature variation due to thermoelastic effect (loading amplitude of $6 \mathrm{kN}$ ): (a1) $5 \mathrm{~mm}-$ notch, (b1) $2 \mathrm{~mm}-$ notch, (c1) $0.7 \mathrm{~mm}$-notch, (d1) $0.5 \mathrm{~mm}$-notch, (e1) $0.2 \mathrm{~mm}$-notch, (f1) $0.1 \mathrm{~mm}$-notch.

In particular 400th-405th cycles: (a2) 5mm-notch, (b2) 2 mm-notch, (c2) $0.7 \mathrm{~mm}$-notch, (d2) $0.5 \mathrm{~mm}$-notch, (e2) $0.2 \mathrm{~mm}$-notch, (f2) $0.1 \mathrm{~mm}$-notch. 


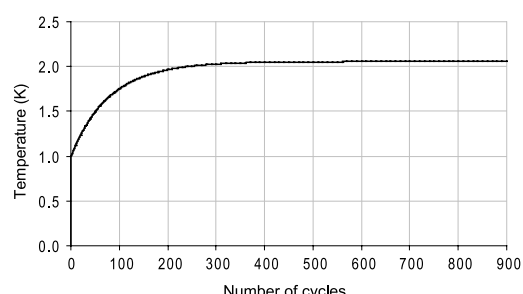

(a1)

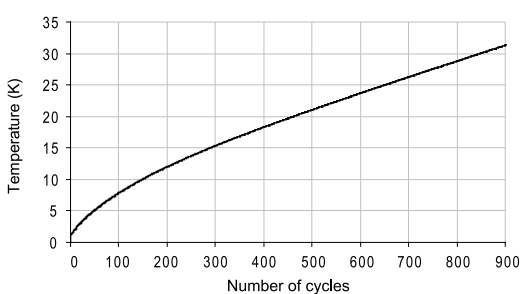

(b1)

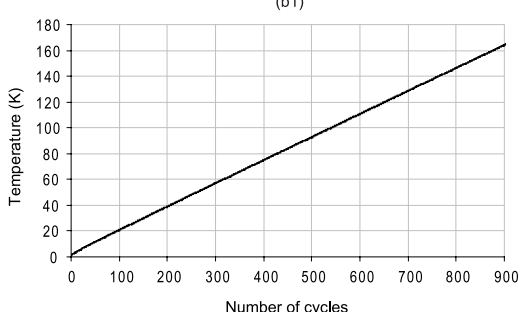

(c1)

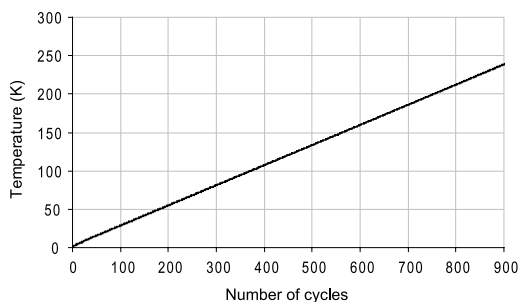

(d1)
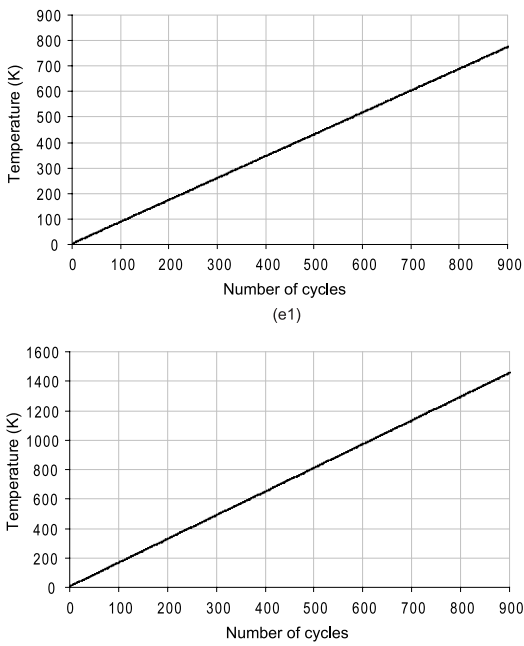

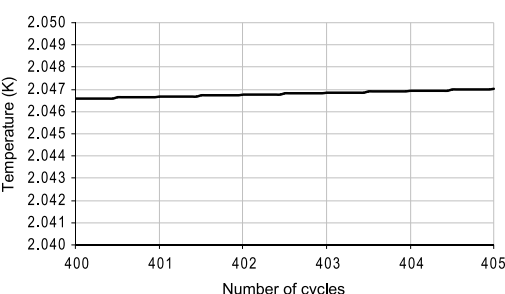

(a2)

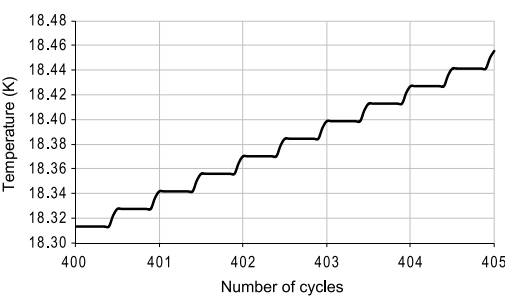

(b2)
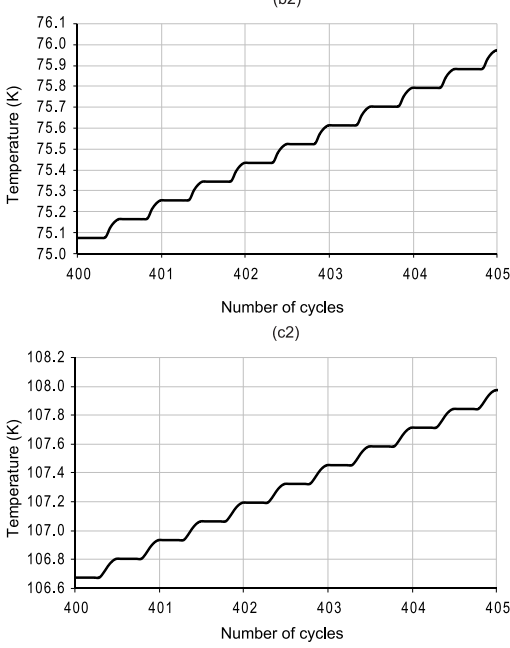

(d2)
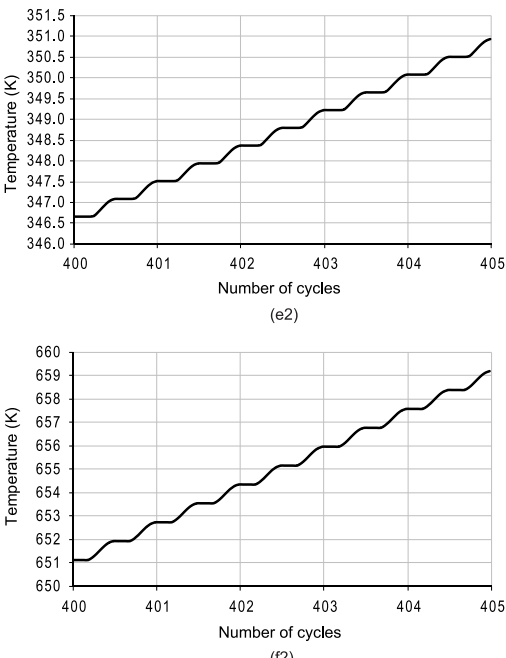

Fig. 6 Temperature increase due to irreversible plastic energy dissipation (loading amplitude of $6 \mathrm{kN}$ ): (a1) 5mm-notch, (b1) 2mm-notch, (c1) $0.7 \mathrm{~mm}$-notch, (d1) $0.5 \mathrm{~mm}$-notch, (e1) $0.2 \mathrm{~mm}$-notch, (f1) $0.1 \mathrm{~mm}$-notch.

In particular 400th - 405th cycles: (a2) 5mm-notch, (b2) 2mm-notch, (c2) $0.7 \mathrm{~mm}$-notch, (d2) $0.5 \mathrm{~mm}$-notch, (e2) $0.2 \mathrm{~mm}$-notch, (f2) $0.1 \mathrm{~mm}$-notch. 

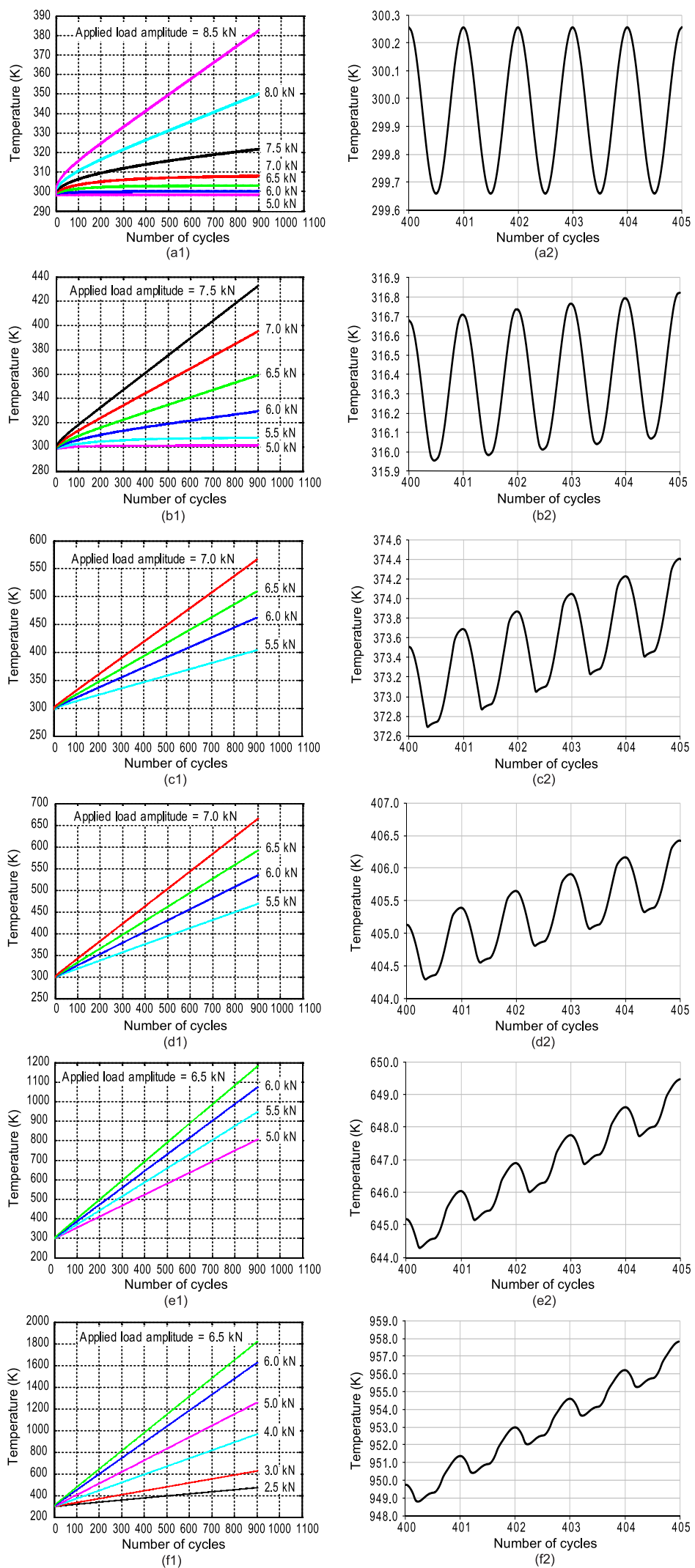

Fig. 7 Temperature evolution during fatigue process: (a1) $5 \mathrm{~mm}-$ notch, (b1) $2 \mathrm{~mm}$ notch, (c1) $0.7 \mathrm{~mm}$-notch, (d1) $0.5 \mathrm{~mm}-$ notch, (e1) $0.2 \mathrm{~mm}$-notch, (f1) $0.1 \mathrm{~mm}$ notch.

400th - 405th cycles in case of loading amplitude of $6 \mathrm{kN}$ : (a2) $5 \mathrm{~mm}$-notch, (b2) $2 \mathrm{~mm}$-notch, (c2) $0.7 \mathrm{~mm}$-notch, (d2) $0.5 \mathrm{~mm}$-notch, (e2) $0.2 \mathrm{~mm}$-notch, (f2) $0.1 \mathrm{~mm}$-notch. 

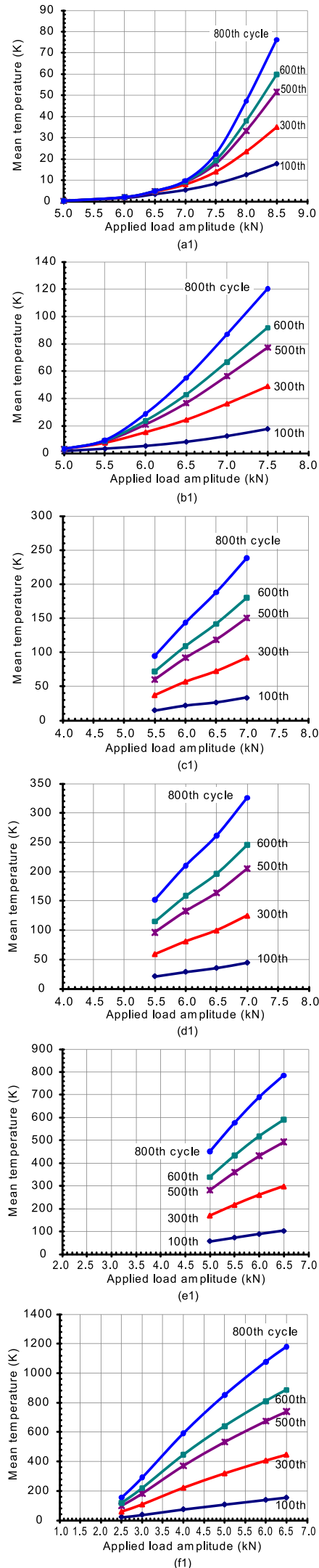

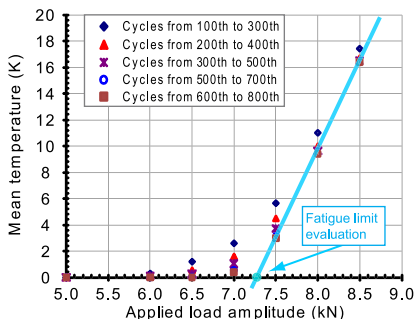

(a2)

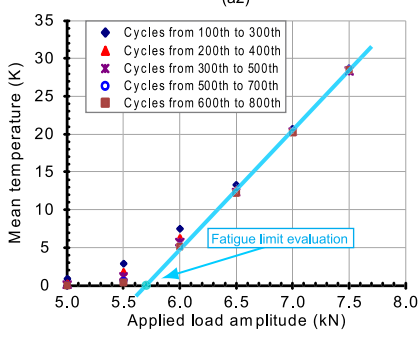

(b2)

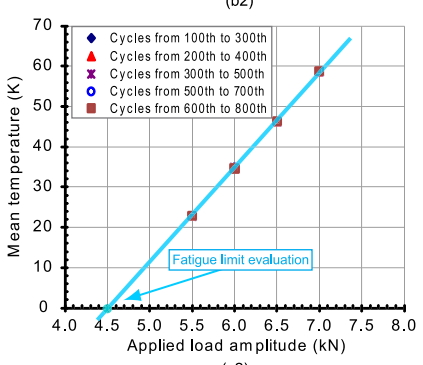

(c2)

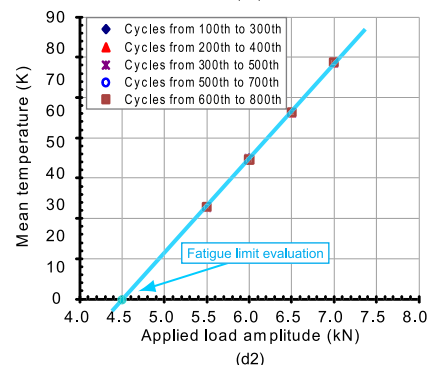

(d2)

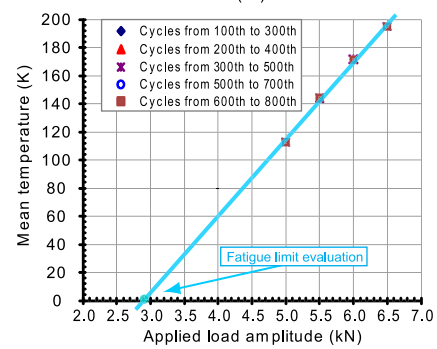

(e2)

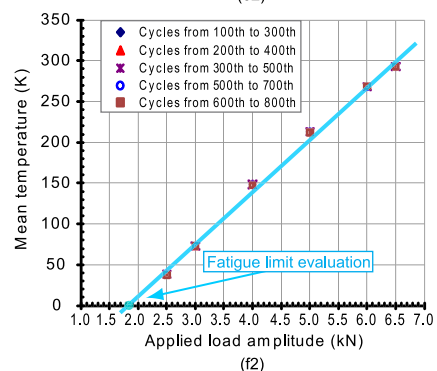

Fig. 8 Mean temperature rise estimation: (a1) 5mm-notch, (b1) 2mm-notch, (c1) $0.7 \mathrm{~mm}-$ notch, (d1) $0.5 \mathrm{~mm}$-notch, (e1) $0.2 \mathrm{~mm}$-notch, (f1) $0.1 \mathrm{~mm}$-notch.

Fatigue limit evaluation:(a2) 5mm-notch, (b2) $2 \mathrm{~mm}$-notch, (c2) $0.7 \mathrm{~mm}-$ notch, (d2) $0.5 \mathrm{~mm}-$ notch, (e2) $0.2 \mathrm{~mm}$-notch, (f2) $0.1 \mathrm{~mm}$-notch. 
are shown in the left column of Fig. 9. The $2 \mathrm{f}$-component amplitude converges to a constant for smaller notch radius, for larger loading amplitude and at larger number of cycles, namely when the shakedown is achieved. In cases of large notch radius ( $5 \mathrm{~mm}$ and $2 \mathrm{~mm}$ ) and for lower loading amplitude, the convergent value is zero, which means the elastic shakedown. By rearranging the data, the $2 \mathrm{f}$-component amplitudes at 100, 300, 500, 600 and 800 cycles were obtained as a function of the loading amplitude as shown in the right column of Fig. 9. The tendency is the same as in the case of the mean temperature rise approach (Fig. 8). The fatigue limit was evaluated in the same manner as in Fig. 8.

The results of fatigue limit evaluation are summarized in Fig. 10 in which the experimental results shown in Fig. 1 are also plotted for comparison. First of all, the fatigue limit obtained by the mean temperature approach matches that obtained by the $2 \mathrm{f}$-component approach for all stress concentration factors simulated. It is reconfirmed that these two approaches are basically equivalent each other under adiabatic condition. In addition, the fatigue limit obtained by numerical simulation coincides with the true value obtained by the Wöhler method very well except for the sharpest notch. The discrepancy at the sharpest notch cannot be explained sufficiently but possible reasons are meso- or micro-structural mechanisms of the fatigue process or heat transfer, both of which are neglected in the present simulation. Unfortunately, the discrepancy between the results of the Wöhler method and the thermography experiment cannot be explained here.

\subsection{Spatial resolution of thermography}

The reason for the discrepancy between the Wöhler method and the thermography experiment shown in Fig. 1 was investigated further. The spatial resolution of the thermography on the specimen surface was about $0.3-\mathrm{mm}$ square in the experiment by Irie et al. ${ }^{(5)}$. The left column of Fig. 11 illustrates the notch, an apparent pixel area (0.3-mm square area) and the finite element meshes used in the simulation for notch radius smaller than $2 \mathrm{~mm}$. It is easily understood that the pixel size is larger than the notch radius and much larger than the mesh size for 0.1 -and $0.2-\mathrm{mm}$ notches.

In order to obtain realistic temperature evolution that should be detected by the thermography in the experiment, average temperature evolution over all elements covered by the pixel illustrated in Fig. 11 (pink square) was computed. As a result, the mean temperature rise and the $2 \mathrm{f}$-component amplitude were obtained as shown in the middle and right columns, respectively. It is confirmed again that these two approaches are basically equivalent under adiabatic condition.

The results of fatigue limit evaluation from middle and right columns of Fig. 11 are also plotted in Fig. 10. The results obtained with considering the spatial resolution becomes larger than those without considering the spatial resolution, which is more remarkable for sharper notches. Of interest is that the fatigue limit evaluated from the numerical simulation becomes closer to that obtained by the thermography experiment if the special resolution is taken into account. Therefore, the discrepancy between the Wöhler method and the thermography experiment can be attributed mainly to the spatial resolution of the thermography.

However, some discrepancy still remains when the stress concentration factors are 4.8 and 6.3. It could be explained as follows. The pixel is not necessarily at the position illustrated in the left column of Fig. 11 but at some shifted position in practice. If the pixel is not at the notch tip, the temperature evolution detected by the thermography would become smaller, and hence both the mean temperature rise and the $2 \mathrm{f}$-component amplitude would become smaller than those shown in Fig. 11. As a result, the least squares fit line (light blue line) moves toward right in Fig. 11 and hence the fatigue limit would be evaluated still larger.

Therefore, the spatial resolution of the thermography should be kept fine enough by considering the stress distribution in the testing object in order to evaluate the fatigue limit correctly. 

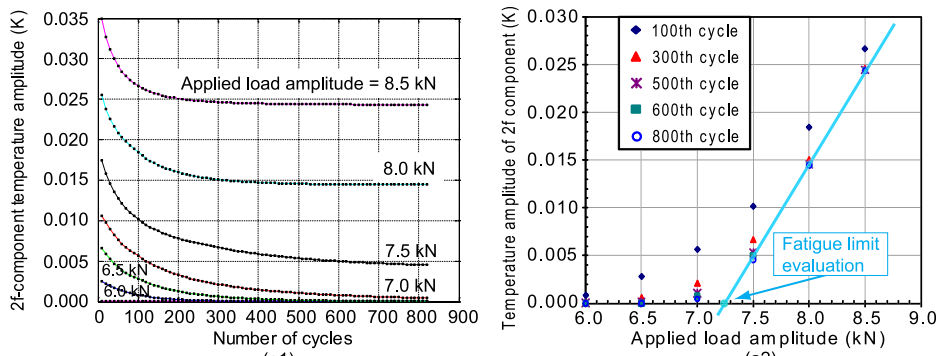

(a1)

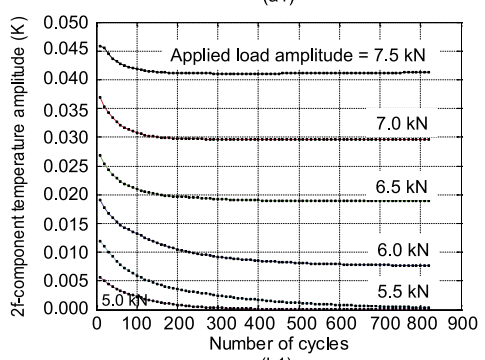

(a2)
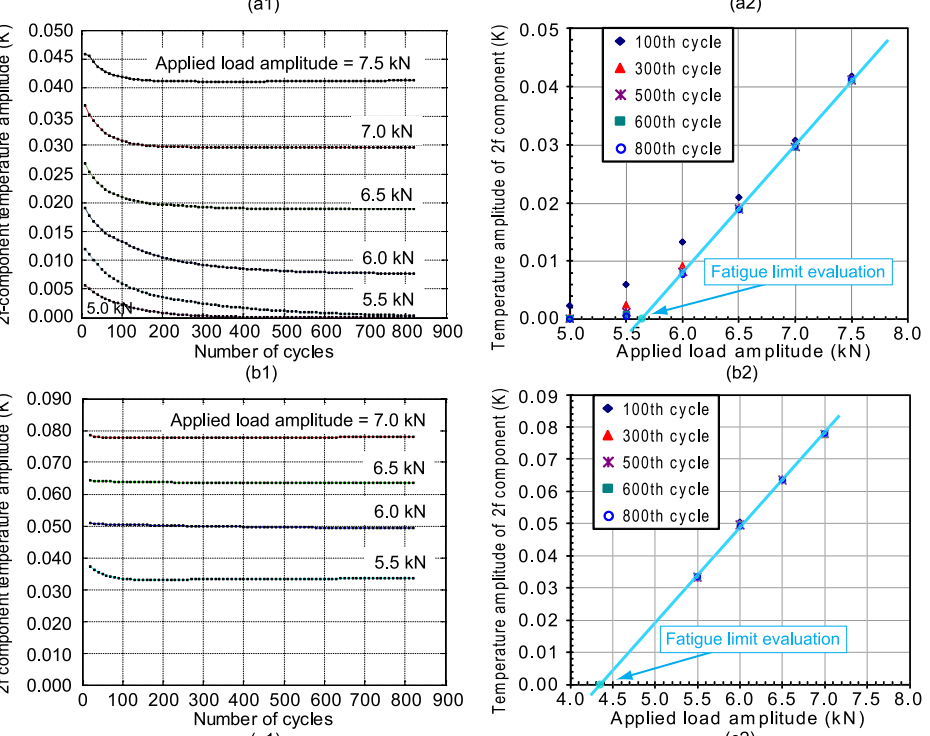

(c1)

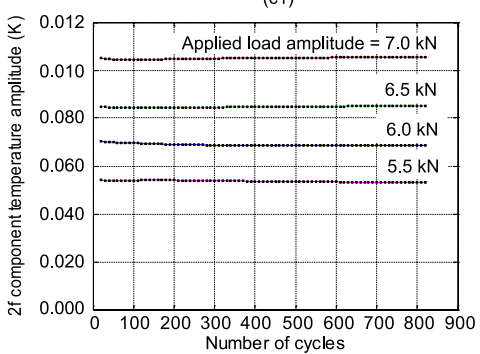

(c2)

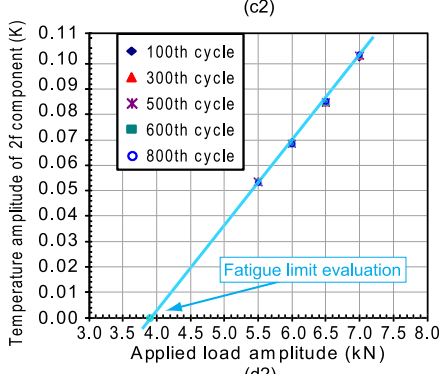
(d1)

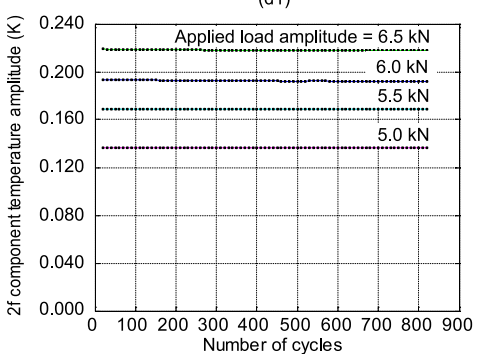

$(\mathrm{e} 1)$

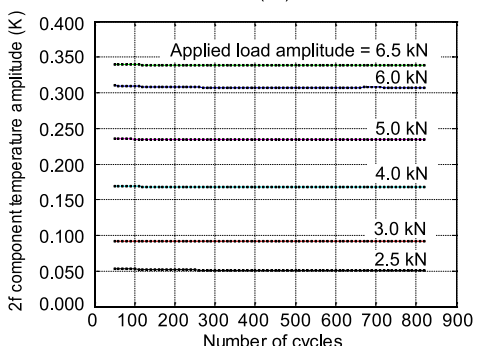

(f1)

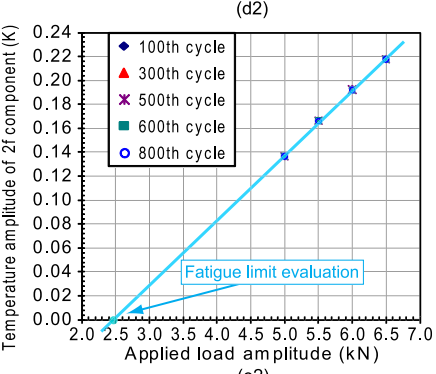

(e2)

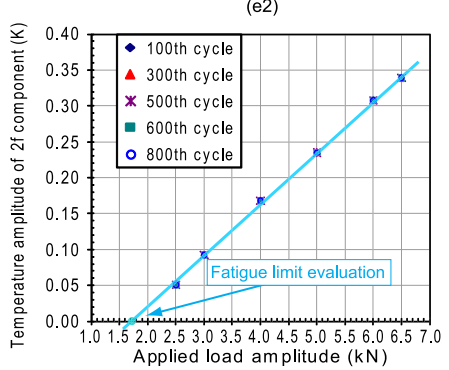

(โ2)

Fig. 9 Temperature amplitude of 2f-component: (a1) 5mm-notch, (b1) 2mm-notch, (c1) $0.7 \mathrm{~mm}$-notch, (d1) $0.5 \mathrm{~mm}$-notch, (e1) $0.2 \mathrm{~mm}$-notch, (f1) $0.1 \mathrm{~mm}$-notch. Fatigue limit evaluation:(a2) $5 \mathrm{~mm}$-notch, (b2) $2 \mathrm{~mm}$-notch, (c2) $0.7 \mathrm{~mm}$-notch, (d2) $0.5 \mathrm{~mm}$-notch, (e2) $0.2 \mathrm{~mm}$-notch, (f2) $0.1 \mathrm{~mm}$-notch. 


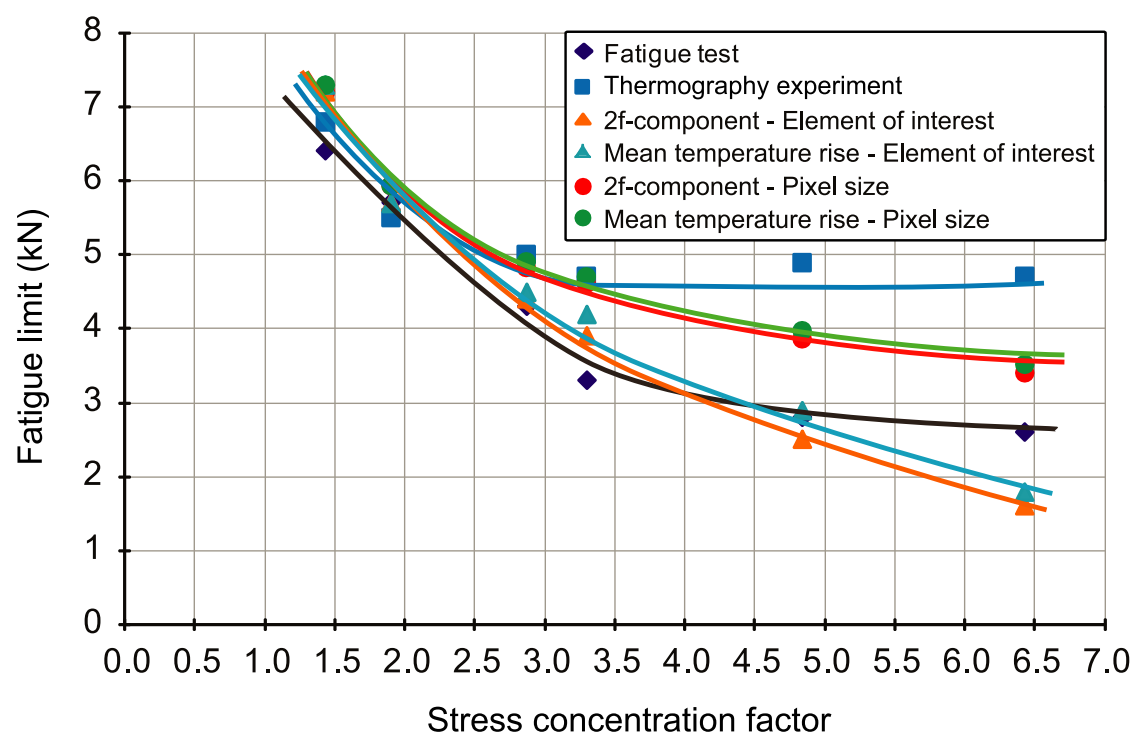

Fig. 10 Fatigue limit evaluation vs. stress concentration factors.

\section{Concluding Remarks}

In this study, 3D elasto-plastic numerical simulation has been conducted for notched specimens with different stress concentration factors in order to clarify the applicability of the technique for rapidly evaluating the fatigue limit to stress concentration problem. The conclusions are summarized as follows.

(1) The conclusions of the previous paper ${ }^{(2)}$ have been reconfirmed for specimens of different stress concentration factors. Namely, (i) the fatigue limit evaluation based on the temperature evolution is essentially explained by plastic energy dissipation, and (ii) the temperature evolution should be measured after a sufficiently large number of cycles so that plastic shakedown is achieved, and in addition (iii) $2 \mathrm{f}$-component and mean temperature rise approaches are basically the same under adiabatic condition.

(2) In order to evaluate the fatigue limit accurately, the spatial resolution of infrared thermography should be fine enough to measure the temperature evolution at the stress concentration part. Otherwise, the fatigue limit is overestimated.

The results of this study can be important guidelines for application of the rapid evaluation of fatigue limit using thermography. However, there remains several issues to be studied.

As mentioned in the previous paper ${ }^{(2)}$, the temperature evolution obtained by numerical simulation is larger than experimental result especially for larger loading amplitude. This should be due to the assumption of adiabatic condition. The temperature distribution within the specimen should be affected by heat transfer, such as heat conduction within the specimen as well as heat convection and radiation to the surroundings among which heat conduction within the specimen would be most important for metallic materials. The heat transfer should affect severely on mean temperature rise which is a consequence of accumulation of heat generated by the plastic energy dissipation. On the other hand, the 2f-component of the temperature evolution would not be affected so much especially when the loading frequency is high enough because the 2f-component corresponds to the heat generated in each cycle. Further study is needed in order to clarify the effect of heat transfer on the fatigue limit evaluation.

In addition, temperature increase for loading amplitude lower than fatigue limit could not be simulated in this paper. The energy dissipation within elastic regime due to nonlinear elasticity or viscoelasticity should be considered for more realistic simulation. Also some kind of size-dependent modeling (for example, modeling of mesoscopic and/or microscopic structures of materials) should be introduced to consider the size effect. 

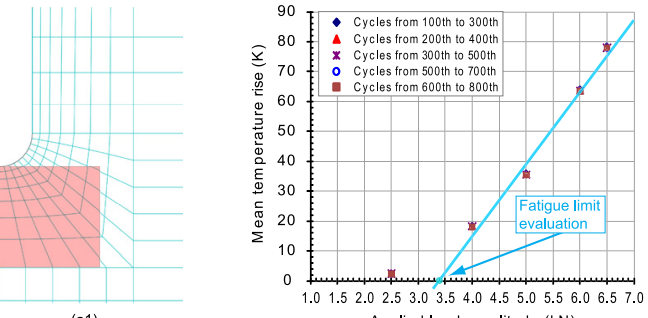

Applied load am plitude $(\mathrm{kN})$

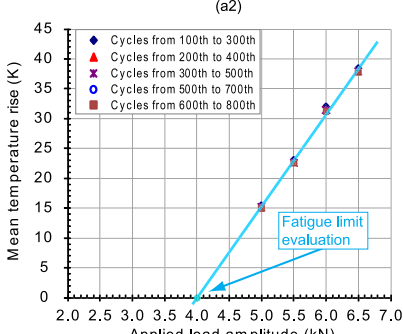

Applied load am plitude $(\mathrm{kN})$

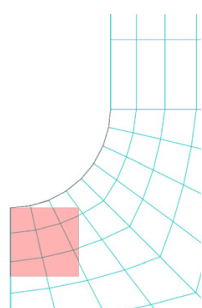

(c1)
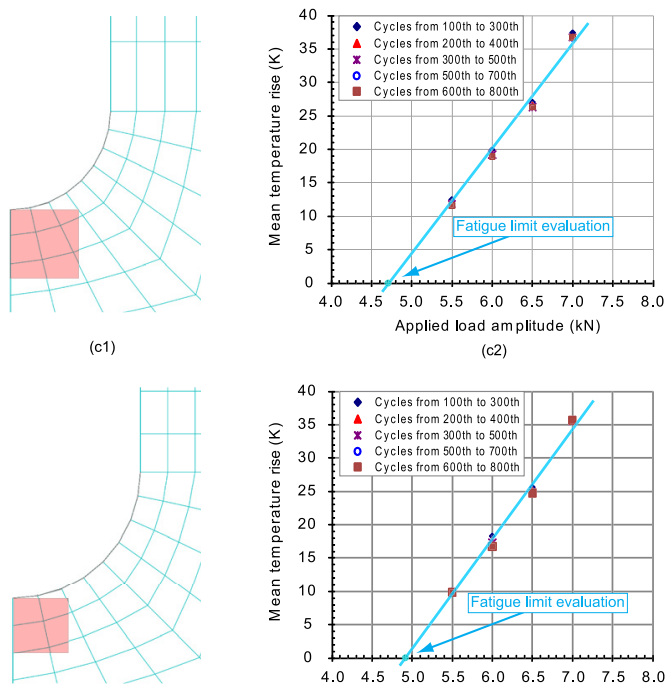

Applied load am plitude $(\mathrm{kN})$

(d2)

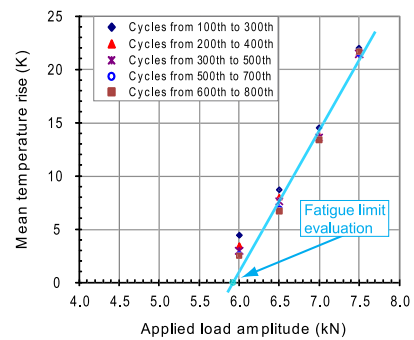

(e2)

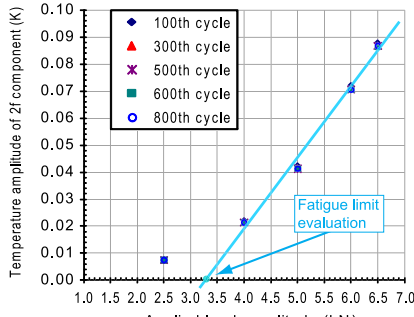

Applied load am plitude $(\mathrm{kN})$
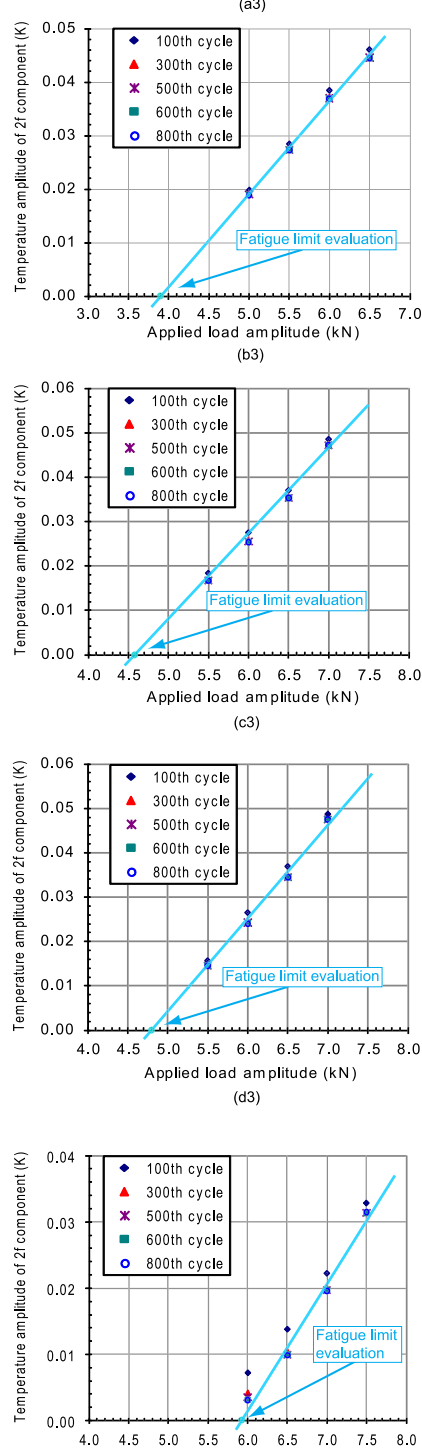

Applied load am plitude $(\mathrm{kN})$

(e3)

Fig. 11 Pixel size compared to element size of notched specimen with radius: (a1) $0.1 \mathrm{~mm}$, (b1) $0.2 \mathrm{~mm}$, (c1) $0.5 \mathrm{~mm}$, (d1) $0.7 \mathrm{~mm}$, (e1) $2.0 \mathrm{~mm}$.

Fatigue limit evaluation by mean temperature rise approach of notched specimen with radius: (a2) $0.1 \mathrm{~mm},(\mathrm{~b} 2) 0.2 \mathrm{~mm}$, (c2) $0.5 \mathrm{~mm}$, (d2) $0.7 \mathrm{~mm}$, (e2) $2.0 \mathrm{~mm}$.

Fatigue limit evaluation by $2 \mathrm{f}$-component approach of notched specimen with radius: (a3) $0.1 \mathrm{~mm}$, (b3) $0.2 \mathrm{~mm}$, (c3) $0.5 \mathrm{~mm}$, (d3) $0.7 \mathrm{~mm}$, (e3) $2.0 \mathrm{~mm}$. 


\section{References}

( 1 ) Vitovec, F.H. and Lazan, B.J., Review of previous work on short-time tests for predicting fatigue properties of materials, Wright Air Development Center Technical Report, No.53-122 (1953), United States Air Force.

( 2 ) Ly, H.A., Inoue, H., and Irie, Y., Numerical simulation on rapid evaluation of fatigue limit through temperature evolution, Journal of Solid Mechanics and Materials Engineering, Vol.5, No.9 (2011), pp.459-475.

( 3 ) Curti, G., La Rosa, G., Orlando, M. and Risitano, A., Analisi tramite infrarosso termico della temperatura limite in prove di fatica, Proceedings, XIV Convegno Nazionale AIAS, (1986), pp.211-220.

( 4 ) Luong, M.P., Infrared thermography of fatigue in metals, Proceedings of SPIE, Vol.1682 (1992), pp.222-233.

( 5 ) Irie, Y., Inoue, H., Mori, T. and Takao, M., Evaluation of fatigue limit of notched specimen by measurement of dissipated energy, Transactions of the Japan Society of Mechanical Engineers, Series A, Vol.76, No.764 (2010), pp.410-412.

( 6 ) Pilkey, W.D., Peterson's Stress Concentration Factors, 2nd Edition, (1997), John Wiley \& Sons.

( 7 ) Dunne, F. and Petrinic, N., Introduction to Computational Plasticity, (2007), Oxford University Press.

( 8 ) Pitarresi, G. and Patterson, E.A., A review of the general theory of thermoelastic stress analysis, Journal of Strain Analysis for Engineering Design, Vol.38, No.5 (2003), pp.405-417.

(9) Biot, M.A., Thermoelasticity and irreversible thermodynamics, Journal of Applied Physics, Vol.27, No.3 (1956), pp.240-253.

(10) Brémond, P., La thermographie infrarouge pour voir les contraintes, Mesures, No.673 (1995), pp.53-56.

(11) Krapez, J.-C., Pacou, D. and Bertin, C., Application of lock-in thermography to rapid evaluation of fatigue limit in metals, Proceedings of the 5th International Workshop on Advanced Infrared Technology and Applications, (1999), pp.379-385. 